MUSIK IN BADEN-WÜRTTEMBERG 


\title{
MUSIK \\ IN BADEN-WÜRTTEMBERG
}

Jahrbuch 1999 / Band 6

\author{
Im Auftrag \\ der \\ Gesellschaft für Musikgeschichte \\ in Baden-Württemberg \\ herausgegeben von \\ Georg Günther und Reiner Nägele
}




\title{
GESELLSCHAFT FÜR MUSIKGESCHICHTE IN BADEN-WÜRTTEMBERG E.V. \\ Schulberg 2, 72070 Tübingen
}

Präsident: Hermann Fünfgeld

Vorsitzender des Wissenschaftlichen Beirats: Prof. Dr. Manfred Hermann Schmid

\section{Anschrift der Redaktion:}

Georg Günther M.A., Otto-Reiniger-Straße 54, 70192 Stuttgart, Tel.: 0711 / 251047

oder

Dr. Reiner Nägele, Württembergische Landesbibliothek, Postfach 1054 41,

Tel.: 07 11 / 212-4441

\author{
Die Deutsche Bibliothek - CIP-Einheitsaufnahme \\ Musik in Baden-Württemberg : Jahrbuch ... / im Auftr. \\ der Gesellschaft für Musikgeschichte in Baden-Württemberg hrsg. \\ - Stuttgart ; Weimar : Metzler. \\ Erscheint jährl. - Aufnahme nach Bd. 1. 1994
}

Bd. 1. 1994 -

\author{
ISBN 978-3-476-01713-0 \\ ISBN 978-3-476-03791-6 (eBook) \\ DOI 10.1007/978-3-476-03791-6
}

Dieses Werk einschließlich aller seiner Teile ist urheberrechtlich geschützt. Jede Verwertung außerhalb der engen Grenzen des Urheberrechtsgesetzes ist ohne Zustimmung des Verlages unzulässig und strafbar. Das gilt insbesondere für Vervielfältigungen, Übersetzungen, Mikroverfilmungen und die Einspeicherung und Verarbeitung in elektronischen Systemen.

Für den Inhalt der einzelnen Beiträge einschließlich Abbildungen sind die Verfasser verantwortlich.

(C) 1999 Springer-Verlag GmbH Deutschland

Ursprünglich erschienen bei J. B. Metzlersche Verlagsbuchhandlung und Carl Ernst Poeschel Verlag GmbH in Stuttgart 1999 


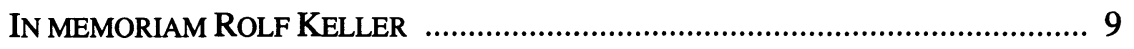

VORBEMERKUNG DER REDAKTION ........................................................... 11

KARL-HERMANN SCHLAGE

Von den Anfängen der evangelischen Kirchenmusik in Mannheim

MiCHAEL GERHARD KAUFMANN

»... im Südwesten des Reichs Träger und Künder deutschen Gesistes ...« -

Die »Staatliche Hochschule für Musik Karlsruhe am Rhein« und ihr

Direktor Franz Philipp

LYDIA JESCHKE

Musik und Technik - Die Donaueschinger Musiktage als Probebühne für neue Medien

PAUL WIEBE

To adorn the groom with chaste delights - Tafelmusik at the weddings of

Duke Ludwig of Württemberg (1585) and Melchior Jäger (1586)

ANDREAS TRAUB

Zur Motette »Miserere mei Deus« von Balduin Hoyoul

DAGMAR GOLLY-BECKER

$\mathrm{Zu}$ den Lebensdaten von Sigmund Hemmel

RÜDIGER THOMSEN-FÜRST

Arkadien am Oberrhein - Zur Aufführungs- und Überlieferungsgeschichte von Joseph Aloys Schmittbaurs Singspiel »Lindor und Ismene«

MATTHIAS WIEGANDT

Dissoziation und Integration - Über Joachim Raffs »Macbeth«

MANFRED HERMANN SCHMID

Schrift der Moderne und Musik der Vergangenheit - Zu Funktionsverschiebungen in der Notations- und Editionspraxis

PETRUS EDER OSB

Einen Konsens aufgekündigt? - Editionsprobleme am Beispiel des ersten

Bandes der »Denkmäler der Musik in Baden-Württemberg«

MANFRED HERMANN SCHMID

Zur Edition von Musik des 16. Jahrhunderts - Formen und Aufgaben historischer Partituren

HELMUT VÖLKL

Neue und restaurierte Orgeln in Baden-Württemberg 1998

Die Autoren der Hauptbeiträge 


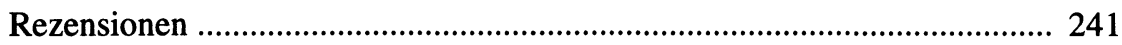

Berichte aus den Musikabteilungen der Landesbibliotheken

Karlsruhe und Stuttgart ...................................................................... 265

Landesmusikbibliographie Baden-Württemberg 1997 ................................. 269

GESELLSCHAFT FÜR MUSIKGESCHICHTE IN BADEN-WÜRTTEMBERG E.V.

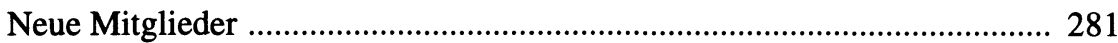

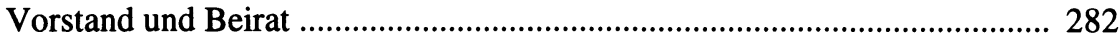

REGISTER

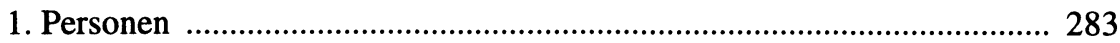

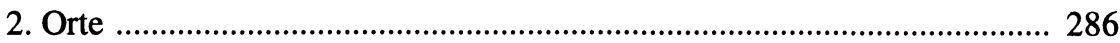


Am 11. September 1998 ist Professor Dr. Rolf Keller, als Ministerialdirektor Amtschef des Justizministeriums in Stuttgart, nach langer und schwerer Krankheit, die er klaglos ertragen hat, im Alter von 63 Jahren in Hechingen verstorben.

$\mathrm{Zu}$ seinem Nachfolger als Präsident der »Gesellschaft für Musikgeschichte in Baden-Württemberg e.V.« wurde auf einer außerordentlichen Mitgliederversammlung am 7. Dezember 1998 in Stuttgart Senator eh. und Intendant i. R. Hermann Fünfgeld gewählt, zum Vizepräsidenten Prof. Dr. Manfred Hermann Schmid. 


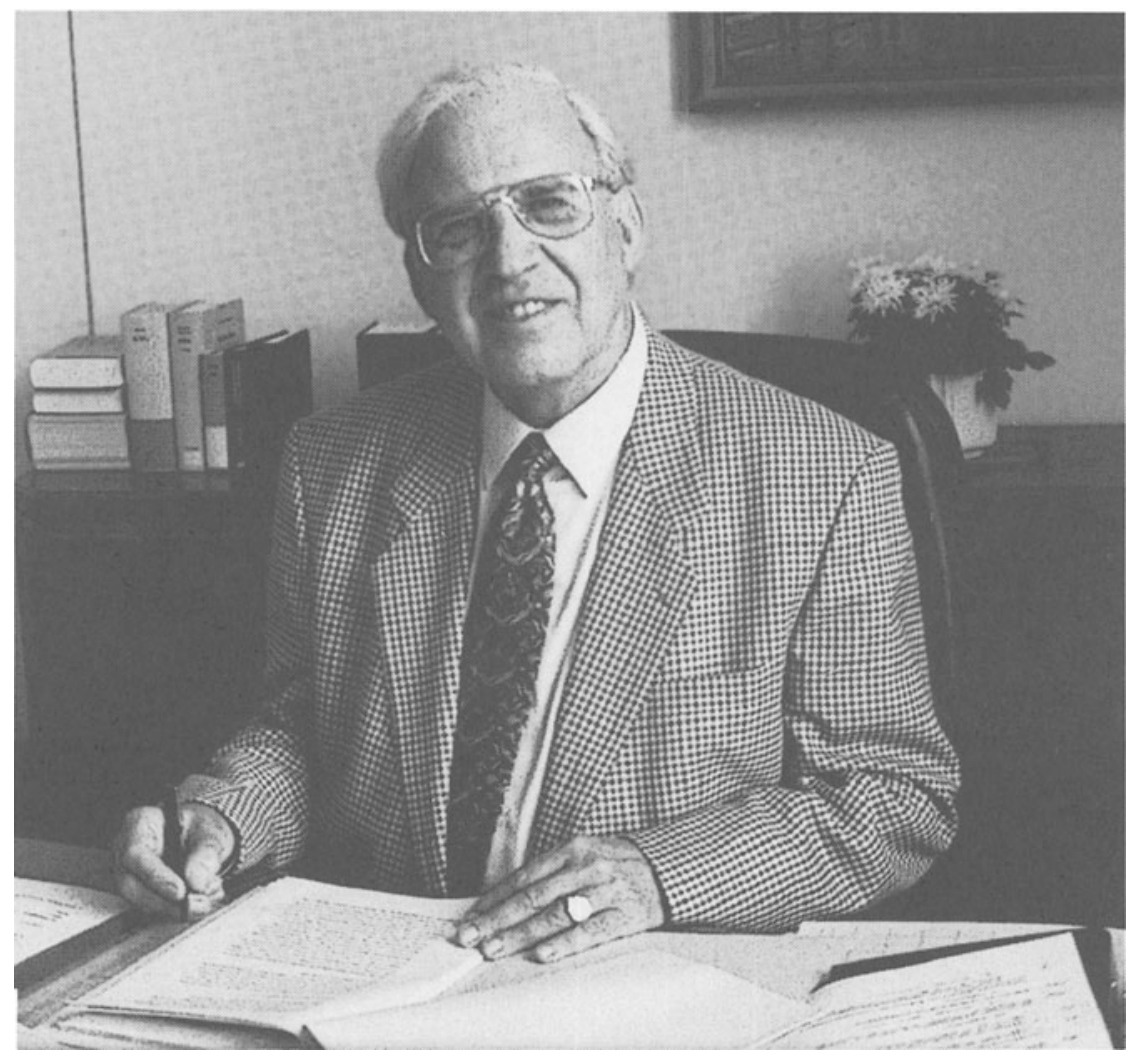

\section{Rolf Keller}

* 19. 6. 1935 - † 11. 9.1998 


\section{In memoriam Rolf Keller}

Wer Rolf Keller gekannt hat, weiß, daß er nicht nur ein glänzender Jurist war, sondern auch ein hochgebildeter Mann, ein Mann der Musen. Unter den Künsten war es die Musik, die ihm geradezu ein Lebenselixier darstellte. Seine ganz besondere Bewunderung galt dem Komponisten Anton Bruckner. In sein Werk hat er sich nicht nur hörend vertieft, sondern es auch studiert und sich sein Umfeld erschlossen. Er war bei allen seinen vielen Aufgaben ein regelmäßiger Besucher der Bruckner-Tage in Linz - und er wäre nicht der gründliche Arbeiter gewesen, als den wir ihn gekannt haben, wenn er bei dieser Gelegenheit nicht auch selbst Forschungsbeiträge vorgestellt hätte.

Die Musik war eine sehr persönliche Vorliebe von Rolf Keller. Aber er hat diese Begabung und Neigung nicht nur privat für sich behalten, sondern auch in den Dienst der Gemeinschaft gestellt. Eines seiner Ehrenämter, und ich glaube, dasjenige, das ihm von allen besonders am Herzen lag, war die Leitung unserer »Gesellschaft für Musikgeschichte in Baden-Württemberg «. Als es nach intensiven Vorgesprächen so weit war, diese Gesellschaft ins Leben zu rufen, am 30. April 1993, wurde Rolf Keller ihr Gründungspräsident. Und er hat die Geschicke der Gesellschaft bis ins seine letzten Tage hinein verfolgt und bestimmt.

$\mathrm{Da} B$ diese Gesellschaft in den vergangenen Jahren aufblühen und ihre Vorhaben so erfolgreich verwirklichen konnte, verdanken wir der Präsidentschaft von Rolf Keller und seinen ungewöhnliche Gaben, seiner Fähigkeit zu persönlicher Anteilnahme, seinem heiteren Ernst und seiner unbedingten Aufrichtigkeit. Diese Eigenschaften haben sich zu dem verbunden, was viele Gesprächspartner immer wieder die unbedingte Integrität Rolf Kellers nannten. Aus ihr erwuchs jene Überzeugungskraft, der wir so viel verdanken.

Rolf Keller ist es gelungen, auseinanderstrebende regionale Kleininteressen zu überwinden und die Arbeit an der Musikgeschichte unseres Landes (und es ist mir unvergeßlich, wie schön er das sagen konnte: »unser Land«), die Erforschung also an seiner Musikgeschichte, zu einem überregionalen Unternehmen zu machen, zu einem Unternehmen im Interesse der Landesgeschichte in ihrer Gesamtheit.

Dauerhaftes Identitätsbewußtsein hängt an Geschichte, an Kunst und Kultur. Als Präsident der »Gesellschaft für Musikgeschichte in Baden-Württemberg " hat Rolf Keller deshalb auch eine politische Aufgabe wahrgenommen und auf seine Weise etwas zum Selbstverständnis Baden-Württembergs beigetragen. Und das Wissen, daß ihn hier etwas überleben wird, war ihm vielleicht ein kleiner Trost in seinem schweren Leiden.

Wir vermissen ihn. Und wir werden an ihn denken. 


\section{VORBEMERKUNG DER REDAKTION}

Mit der Edition der lateinischen und deutschen Motetten des Stuttgarter Hofkapellmeisters Balduin Hoyoul legte die Redaktion der »Denkmäler der Musik in Baden-Württemberg « 1998 bereits den siebten Band ihrer Publikationsreihe vor. Da die Diskussion über das zugrundegelegte Editionskonzept in der Fachpresse in den vergangenen Jahren kontrovers geführt wurde, soll mit dem aktuellen Jahrbuch die Möglichkeit geboten werden, sich mit der immer noch einige Irritationen auslösenden Editionspraxis der Denkmälerreihe auseinanderzusetzen. Unterschiedlich Intentionen zeichnen die Beiträge aus: Zum einen stellt Manfred Hermann Schmid die Frage nach der funktionalen Differenz zwischen »Komponier-« und »Notenpultschrift « und den daraus resultierenden Konsequenzen für eine Edition unter modernen technischen Bedingungen. In einem zweiten Beitrag begründet Schmid die Editionsprinzipien der »Denkmäler « anhand editorischer Fragen an das historisch überlieferte Notenbild. Petrus Eder OSB beschäftigt sich hingegen mit der 1995 von Ulrich Siegele an mehreren Stellen vorgebrachten Kritik am Editionskonzept.

Neben diesem thematischen Schwerpunkt befassen sich die drei Artikel, die das Jahrbuch eröffnen, mit städtischer und institutioneller Geschichte unseres Bundeslandes, nämlich zur Kirchenmusik in Mannheim (Karl-Hermann Schlage), zur Staatlichen Hochschule für Musik in Karlsruhe im "Dritten Reich « (Michael Gerhard Kaufmann) sowie zu den Donaueschinger Musiktagen (Lydia Jeschke). Erstmals ist nun auch eine englischsprachige Studie unter den Beiträgen zu finden, nämlich die im Rahmen einer Dissertation entstandene Untersuchung des kanadischen Musikwissenschaftlers Paul Wiebe zu den Tafelmusiken bei den Hochzeitsfeierlichkeiten von Herzog Ludwig von Württemberg und dessen Kammersekretär Melchior Jäger; eine kurze Zusammenfassung in deutscher Sprache findet der Leser am Schluß dieses Beitrages. Andreas Traub widmet sich einer vergleichenden Betrachtung zweier Motetten von Hoyoul und Josquin Desprez. Ergänzungen zur Biographie Sigmund Hemmels liefert der Beitrag von Dagmar Golly-Becker. Zwei Werkbetrachtungen mit unterschiedlicher methodischer Zielsetzung - einmal enstehungs- und aufführungsgeschichtlich, zum anderen analytisch orientiert - ergänzen das Ensemble: Rüdiger Thomsen-Fürst widmet seine Studie Joseph Aloys Schmittbauers Singspiel »Lindor und Ismene«, Matthias Wiegand legt eine sehr detaillierte Untersuchung von Joachim Raffs »Macbeth«-Vertonung vor.

Auch in diesem Band befinden sich Rezensionen, die Landesmusikbibliographie 1998, die Rubrik »Neue und restaurierte Orgeln in Baden-Württemberg" sowie Berichte aus den beiden Landesbibliotheken Karlsruhe und Stuttgart. Inhaltsverzeichnis und Rezensionsteil - auch der früheren Bände - können wie gewohnt als Volltext im Internet unter folgender Adresse abgerufen werden:

http://www.wlb-stuttgart.de/ www/referate/musik/forschung.html 